\title{
0317 WHAT PROTECTS YOUNGSTERS FROM VIOLENCE
} VICTIMISATION?

R Gofin, M Avitzour* Correspondence: School of Public Health and Community Medicine, Hadassah and Hebrew University, POB 12000, Jerusalem, 91120, Israel

10.1136/ip.2010.029215.317

Background Violence, in its varied presentation forms is common among youth. Risk factors have been identified in diverse populations for different types of violence. In this study we assessed victimisation for specific types of violence common among young people, and determined what the personal, familial and school protecting factors are.

Methods Students (12-14 years old) in 35 randomly selected junior high schools were from the Jerusalem Hebrew (secular and religious) and Arab educational system ( $n=2610)$. They answered an anonymous questionnaire, addressing personal, family, and school characteristics, unintentional injuries and violence. Victimisation included experiencing at least one of the following: was bullied, mocked, boycotted, was stolen or was taken personal belongings by force.

Results Only $24.2 \%$ did not experience violence in the last term. In a multivariate analysis independent protective factors from victimisation were: type of school, being older, female (OR 1.76, 95\% CI 1.40 to 2.21), being participated in activities (OR 4.33, 95\% CI 2.82 to 6.63), having two or more close 


\section{IP Safety 2010 abstracts}

friends (OR 1.82, 95\% CI 1.07 to 3.10), having good relations among students (OR 2.73, 95\% CI 1.04 to 7.15) and having a high family support (OR $1.53,95 \%$ CI 1.19 to 2.00). Hours of television viewing, participating in sports activities, membership in a youth movement or the reported family socioeconomic status were not significant protective factors.

Conclusions Few children are not victims of violence in school. Protective factors for violence are positive personal, family and school relations. Interventions addressing these positive factors may help to reduce violence in young people and their natural environment. 